\title{
EM Information Security Threats against RO-based TRNGs: The Frequency Injection Attack Based on IEMI and EM Information Leakage
}

\author{
Saki Osuka, Student Member, IEEE, Daisuke Fujimoto, Member, IEEE, Yu-ichi Hayashi, Member, \\ IEEE, Naofumi Homma, Senior Member, IEEE, Arthur Beckers, Josep Balasch, Benedikt Gierlichs, \\ and Ingrid Verbauwhede, Fellow, IEEE
}

\begin{abstract}
True random number generators (TRNGs) based on ring oscillators (ROs) are employed in many devices because they can be constructed with a simple circuit structure. Many systems are affected if an RO-based TRNG is attacked, and its security is degraded. Conventional attacks against RO-based TRNGs reduce randomness using direct physical access to the target device and/or modification/invasion of the device or the equipment on which it is implemented. However, depending on the physical location of the device and its tamper resistance measures, directly accessing the device or operating/modifying the implementation may not be easy. This study introduces a noninvasive attack against RO-based TRNGs. In this attack, we intentionally induce sinusoidal electromagnetic waves in a TRNG and estimate the change in its randomness under this interference by observing the signal leaked from the TRNG from a distance. We also consider countermeasures against noninvasive attacks on TRNGs.
\end{abstract}

Keywords - true random number generator, intentional electromagnetic interference, EM information leakage

\section{INTRODUCTION}

True random number generators (TRNGs) generate random numbers based on a random physical phenomenon. The bit stream of independent bits generated by a TRNG does not reproduce the same sequence (non-repeatability); hence, TRNGs are in demand in the security field. Therefore, any degradation in TRNG randomness is a real threat to security [1] TRNGs based on ring oscillators (ROs) are particularly used in many devices because their circ ${ }^{1}$ uit structure is simple. Many systems are affected when RO-based TRNGs are attacked, and the randomness is reduced.

This work was supported in part by JSPS KAKENHI Grant Number $16 \mathrm{H} 02831$ and in part by JSPS and FWO under the Japan - Belgium Research Cooperative Program.

S. Osuka, D. Fujimoto and Y. Hayashi are with the Nara Institute of Science and Technology, Nara 630-0192, Japan (e-mail: osuka.saki.ok1@is.naist.jp; fujimoto@is.naist.jp; yu-ichi@is.naist.jp).

N. Homma is with the Research Institute of Electrical Communication, Tohoku University, Japan.

A. Beckers, J. Balasch, B. Gierlichs and I. Verbauwhede are with the imecCOSIC group of Departement Elektrotechniek (ESAT), KU Leuven, Belgium.
A physical attack against RO-based TRNGs using frequency injection was proposed by Markettos and Moore [1]. By injecting a continuous wave directly into the power line of the device, they were able to suppress jitter (i.e., the randomness source of the TRNG) and degrade its output. Subsequently, the randomness of the TRNG could be degraded by injecting a continuous wave to the vicinity of the circuit [2], [3]. Likewise, a TERO-based TRNG analogous to an RObased TRNG degraded the randomness by introducing a continuous wave from the vicinity of the circuit [4]. These methods implicitly assume that implementing a direct physical attack that modifies or invades the target device is possible. However, many cryptographic devices are equipped with tamper-resistance features (for instance, a case or a mechanism that detects modification) that make it difficult to physically access or modify the target modules. In addition, some devices are installed in places, where physical access is difficult, and it is difficult to apply this attack method under such circumstances.

A method for intentional electromagnetic interference (IEMI) fault injection from a distance was presented [5]. Using this technique, faults were noninvasively injected to a target device from outside its close environment. Temporal faults can be remotely injected into the target device through the insertion of sinusoidal electromagnetic (EM) waves via power or communication cables attached to the target device. Thus, by employing this method, attacks could be performed even if direct access to the target device is difficult.

This study investigates the possibility of an attack that degrades the randomness of TRNGs when close physical access to them is difficult. Sinusoidal waves are specifically induced in the TRNG via power or communication cables from a distance through frequency sweeping. At the same time, the signal leaked during the TRNG operation is remotely measured, thereby allowing the observation of the fluctuation pattern of the jitter (the source of randomness) and the estimation of whether the randomness of the TRNG has been degraded.

The proposed noninvasive frequency injection method based on IEMI can be performed through any external cable, such as a power or communication cable, and thus, has the potential to be used to attack a wide variety of devices 
equipped with TRNGs. Consequently, countermeasures that account for these types of remote attack should be devised. Unconventional countermeasures that do not assume an intrusive attack must be taken to counter attacks using the proposed method. We also consider countermeasures against non-invasive attacks on TRNGs.

\section{NONINVASIVE FREQUENCY InJeCtion ATtACK}

In a frequency injection attack aimed to degrade the randomness of a TRNG, an injection frequency that can suppress the jitter as a source of randomness must first be selected, then sinusoidal waves must be induced at that frequency in the target TRNG [1]. Analytically obtaining the appropriate frequency is difficult. In the conventional method [1], the frequency is selected by observing the operation of the ROs or obtaining the bit stream generated by the TRNG directly. However, when noninvasively attacking from a distance, finding an appropriate injection frequency is not easy because of the difficulty of observing RO operations or TRNG outputs from outside the device. In addition, when considering attacks on actual devices, an appropriate injection frequency may depend on the individual device because the operation of the ROs is determined by the manufacturing variations of each individual inverter. Even if we could obtain a device that was the same product as the target device and profiled the injection frequency, it would be ineffective to use this profiled injection frequency to attack the target device. Therefore, a method to estimate the randomness of the TRNG using information obtained from a distance is necessary for noninvasive remote frequency injection attacks. Some changes occur in the RO operations when the TRNG randomness is degraded, if these changes can be observed from outside the device; hence, estimating an appropriate injection frequency that degrades this randomness is possible.

As shown in Fig. 1, we propose a noninvasive frequency injection attack against RO-based TRNGs. Potential impedance mismatches are generally in electrical devices; thus, these impedance mismatches will result in degraded immunity and increased emissions at certain frequencies. Therefore, we focus on these frequencies, perform intentional interference and emission measurement on the system, and execute the attack. The sinusoidal EM waves at a specific frequency are introduced into a connection line, such as a power or communication cable attached to the device. The changes in the internal states of the ROs can be measured and the frequency at which the randomness of the TRNG is degraded can be determined by measuring the side-channel information generated by the TRNG at a distance.

This section presents a mechanism to decrease the randomness of the RO-based TRNGs under a frequency injection attack and describe how to estimate the degradation of the randomness using side-channel information.

\section{A. Suppression of jitter in a frequency injection attack}

The basic principle of the widely used RO-based TRNGs was proposed by Sunar et al. [7]. This RO-based TRNG is composed of $\mathrm{r}$ distinct ROs consisting of $\mathrm{n} 1, \ldots, \mathrm{nr}$ inverters (taking arbitrary odd values), an XOR gate, and D flip-flops (Fig. 2). The output signals of each RO are exclusive-ORed,

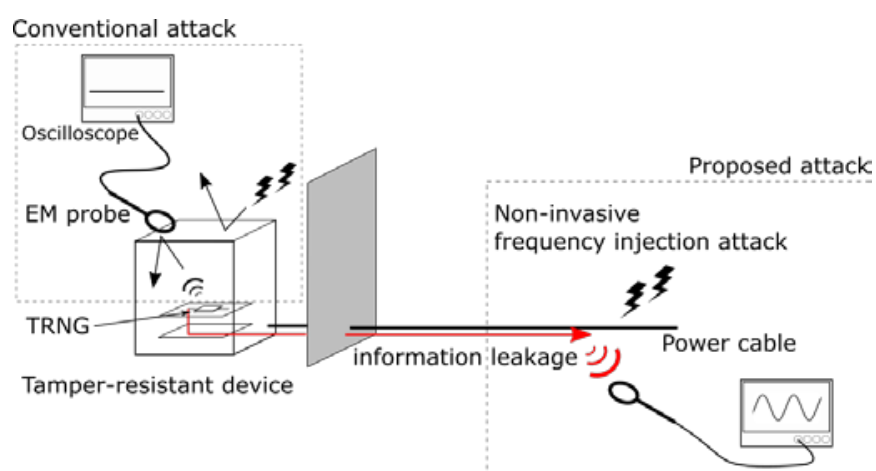

Fig. 1. Noninvasive frequency injection attack flow against an RO-based TRNG

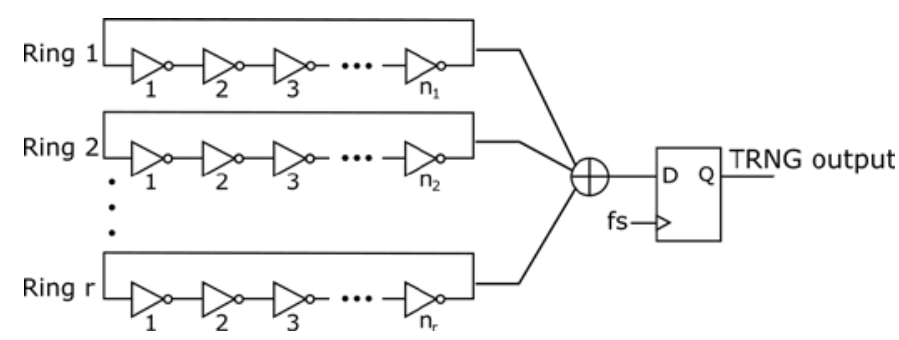

Fig. 2. Basic RO-based TRNG

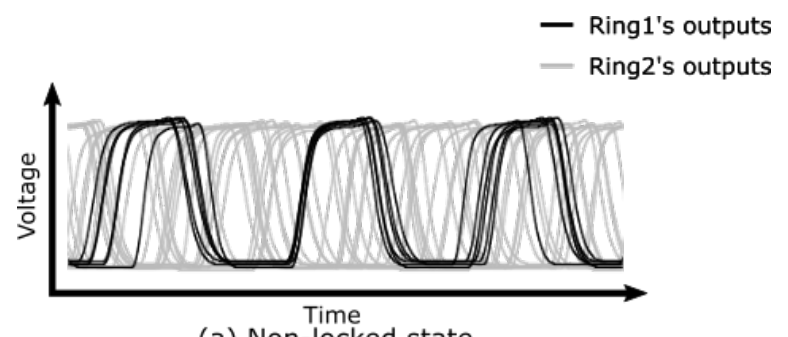

(a) Non-locked state

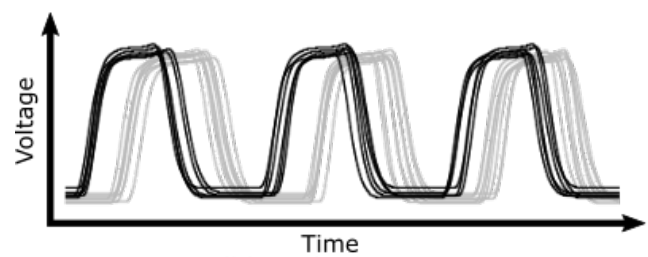

(b) Locked state

Fig. 3. RO waveforms in (a) unlocked and (b) locked states

and the resulting signal is sampled at some uncorrelated frequency $f_{s}$ to produce a stream of bits. The randomness of the bit stream generated by an RO-based TRNG comes from jitter, which is the random noise of the RO. In the time domain, jitter appears as variations in the transition timing of an RO caused by its uncertainty, which causes the transitions to occur before or after the expected switching time. Jitter is cumulative over time, and the accumulated jitter increases the uncertainty in the transition timing; thus, the output fluctuates (Fig. 3(a)). As the jitter accumulates, the output of each RO increases in randomness in a way that is unrelated to the outputs of the other ROs. Consequently, the exclusive-ORed output of all ROs becomes random. 
In a frequency injection attack, a resonator with a certain natural frequency will lock to it when driven by a specific frequency, similar to the resonance phenomenon of a pendulum. Hence, the jitter of each RO becomes locked to the jitter of the injected sinusoidal wave, and the jitter between the ROs becomes synchronized [8]. The synchronization of the jitter suppresses the transition timing variability of each RO, causing the fluctuation of the ROs to decrease (Fig. 3(b)). This fluctuation decreases; hence, the output of each RO correlates with that of the other ROs, causing the randomness of their output pattern to reduce and the randomness of the TRNG to degraded.

\section{B. Observation of common-mode current as signal leakage}

This section outlined the side-channel information that contains information about the RO states and examines the method used to estimate the randomness of a TRNG by observing the common-mode current as the side-channel information noninvasively. First, we explain how the common-mode current can be observed from outside the device to reveal information about the RO states. Next, we outline the method for estimating the reduced randomness of the TRNG.

Unintentional information leakage is well known to occur in the common-mode current, which reflects the internal processing of the devices [9-12]. The transient current caused by the changes in the ROs' internal processing is transformed into a voltage fluctuation through inductance in the circuit. This voltage fluctuation is propagated as a common-mode current to peripheral circuits, for instance, through circuits with a common ground to a power cable. A previous work [13] reported that the common-mode current observed via a power cable can be exploited for EM analysis attacks. The internal processing of the ROs is assumed to generate a common-mode current because of the same principle; hence, there is a high possibility of acquiring information related to the RO states from a power cable.

Figure 4 shows the outline of the proposed method in this study. First, a continuous sinusoidal EM wave at frequency $f_{i n j 1}$ is introduced to a device equipped with a TRNG by an injection probe clamped to a power cable. Subsequently, the common-mode current is measured using a current probe also clamped to the power cable. We can estimate the degradation of the randomness if we are able to acquire the information related to the RO internal state changes caused by the locking of the ROs (Fig. 3) from the common-mode current.

\section{EXPERIMENT}

This section shows the feasibility to inject the attack signals locking the ROs from a distance. The RO states are observed from the side-channel information using the method shown in the previous section. Furthermore, we demonstrate that the randomness of the TRNG can be estimated using only side-channel information.

The injection probe used to induce the sinusoidal waves is commonly used for testing the immunity of various devices, such as in bulk current injection tests [14]. Specifically, when

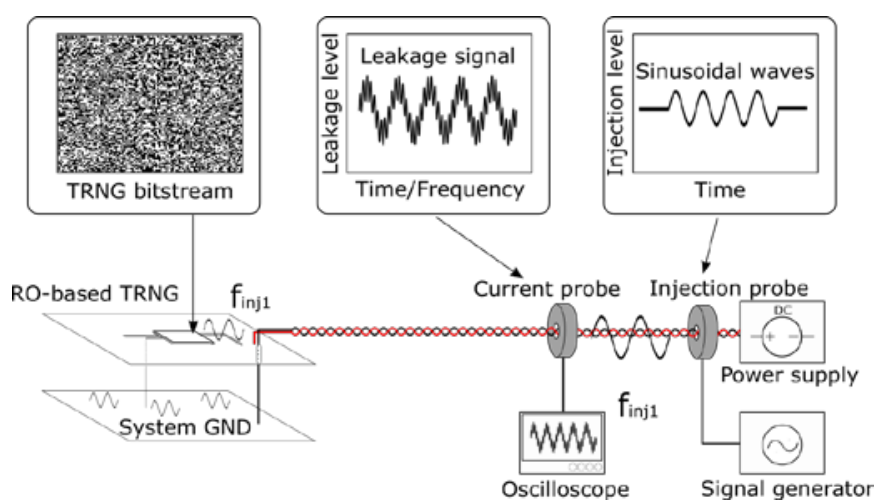

Fig. 4. Noninvasive frequency injection attack on an RO-based TRNG from a distance

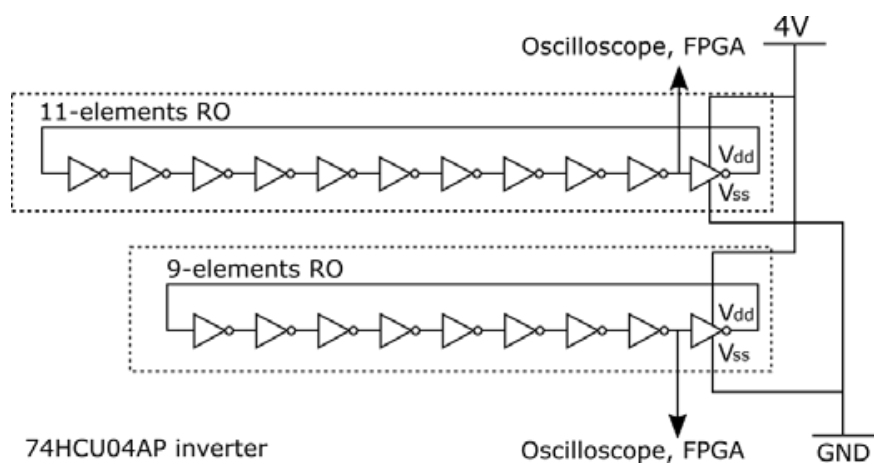

Fig. 5. RO-based TRNG that consists of an 11- and a 9-element RO

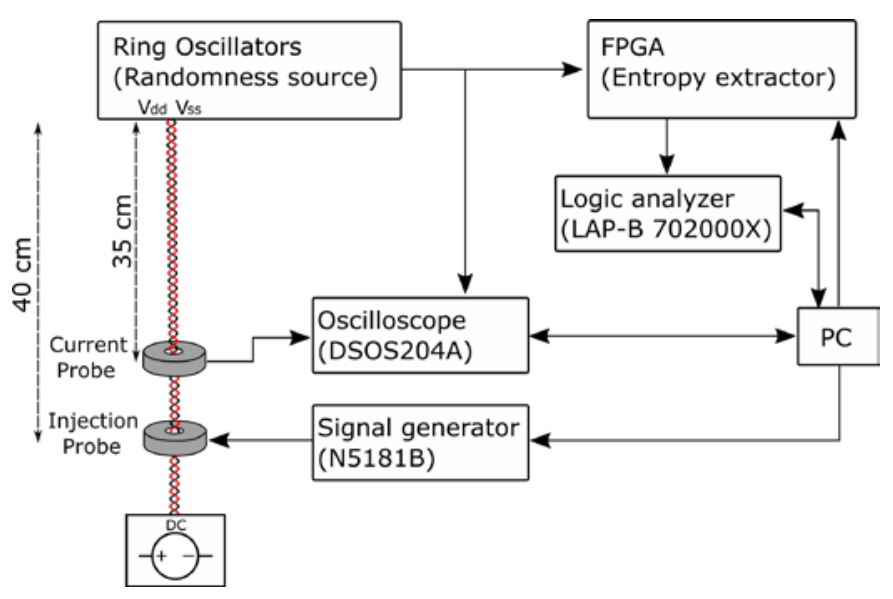

Fig. 6. Experimental setup

Table 1 Equipment used in the experiment.

\begin{tabular}{|c|c|}
\hline Inverter & 74HCU04AP \\
\hline Injection probe & $\begin{array}{c}\text { FCC F-140 } \\
(100 \mathrm{kHz} \text { to } 1.3 \mathrm{GHz})\end{array}$ \\
\hline Current probe & FCC F-2000 \\
& Keysight DSOS204A \\
\hline Oscilloscope & Keysight N5181B \\
\hline Signal generator & Zeroplus LAP-B 702000X \\
\hline Logic analyzer &
\end{tabular}


the injection probe is applied to a cable attached to the target device, disturbance waves can be generated in the cable by the electromagnetic coupling between the injection probe and the cable. In our experiment, a current probe is also clamped to the power cable to observe the side-channel information. In addition, the common-mode current is measured via the power cable using an oscilloscope and a current probe attached to the power cable to confirm the changes in the RO states. Furthermore, an exclusive OR of the RO outputs is taken using the XOR gate implemented in the FPGA, and the output of the exclusive OR is sampled at an arbitrary frequency by the logic analyzer, such that random numbers are generated. We lastly evaluate the randomness of the TRNG.

\section{A. Experimental Setup}

We evaluated an RO-based TRNG that consisted of 11- and 9-element RO as the target device (Fig. 5). We employed the 74HCU04AP inverter chip to construct the rings, and the supply voltage was $4.0 \mathrm{~V}$. The natural frequencies of the 11and 9-stage ROs were approximately 16.27 $\mathrm{MHz}$ and 18.67 $\mathrm{MHz}$, respectively. In the experiment, we attacked the TRNG by injecting disturbance waves and estimating the randomness of the TRNG using side-channel information from outside the device.

Figure 6 and Table 1 show our experimental setup and equipment used in the experiment, respectively. Attack signals that impair the randomness of TRNG were introduced through IEMI. The continuous sinusoidal waves generated by a signal generator were induced by an injection probe into a power cable attached to the TRNG. We observed the common-mode current caused by the internal processing of the ROs using a current probe clamped to the power cable to estimate the randomness of the TRNG. The obtained waveforms were observed with an oscilloscope. We observed the oscilloscope signal to evaluate the locking of the ROs. We obtained the bit stream generated by the TRNG by taking the exclusive OR of the RO outputs from the FPGA and sampling it at $1 \mathrm{kHz}$ with a logic analyzer to obtain the TRNG randomness.

As shown in Fig. 6, the current probe and the injection probe were located approximately 35 and $40 \mathrm{~cm}$ away from the TRNG, respectively.

\section{B. Spectrum change of the common-mode current at frequency injection}

A significant difference in the common-mode current was observed when the amplitude of the injected sinusoidal waves was $25.2 \mathrm{dBm}$, and the injected frequencies were set to 32.3 and $57.0 \mathrm{MHz}$.

Figure 7 shows the RO outputs on the oscilloscope. In the time domain traces, we obtained the RO outputs triggered by the 11-stage RO (black line) and showed 100 overlaid waveforms. The gray line in the background of the plot shows the 9-stage RO outputs. Figure 7(a) shows the result of injecting 32.3 MHz, where the 9-stage RO outputs had many patterns with respect to the 11-stage RO, and the waveforms substantially fluctuated. This result indicated that the transition timings of the 9-stage RO varied with respect to the 11-stage RO, as suggested in Fig. 3(a). Accordingly, the ROs
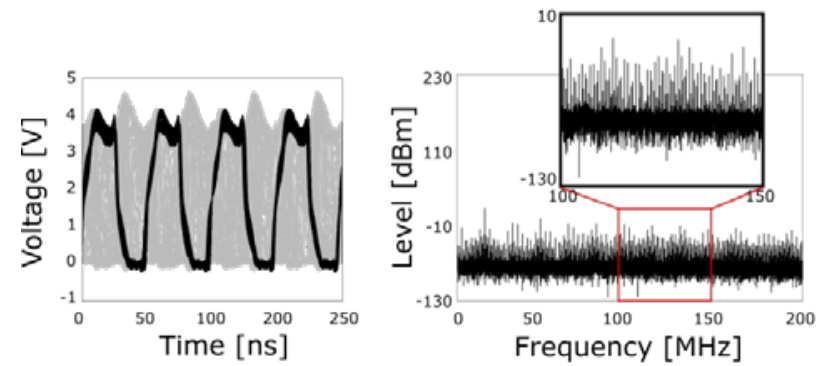

(a) $32.3 \mathrm{MHz}$ injection
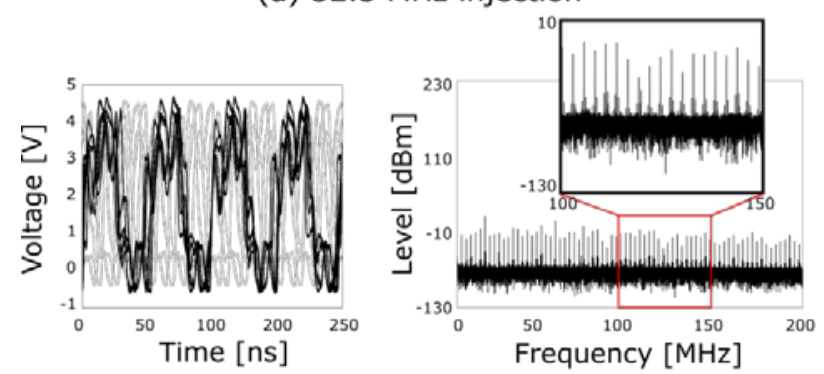

(b) $57.0 \mathrm{MHz}$ injection

Fig. 7. Changes in outputs and frequency spectrums of RO's outputs when under attack
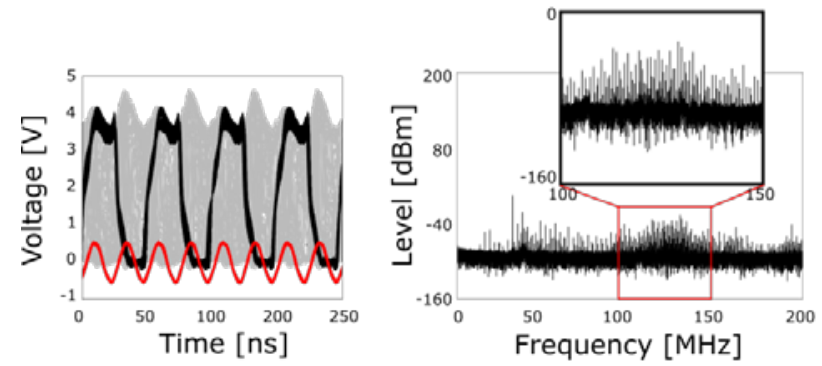

(a) $32.3 \mathrm{MHz}$ injection
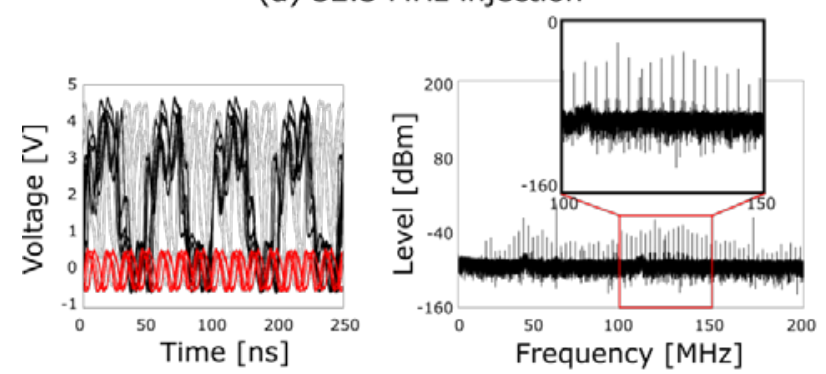

(b) $57.0 \mathrm{MHz}$ injection

Fig. 8. EM information leakage Changes in outputs and frequency spectrums of EM information leakage signal when under attack

were not locked when the sinusoidal waves were injected at 32.3 MHz. In contrast, when 57.0 MHz was injected (Fig. 7(b)), the 9-stage RO outputs clearly had a few patterns with respect to the 11-stage $\mathrm{RO}$, and the fluctuation of waveforms was small. This finding suggested that the variation in the transition timing was reduced by locking the ROs. Hence, these results demonstrated that we can remotely inject attack signals (approximately $40 \mathrm{~cm}$ from the device) and lock the ROs at a certain frequency. 


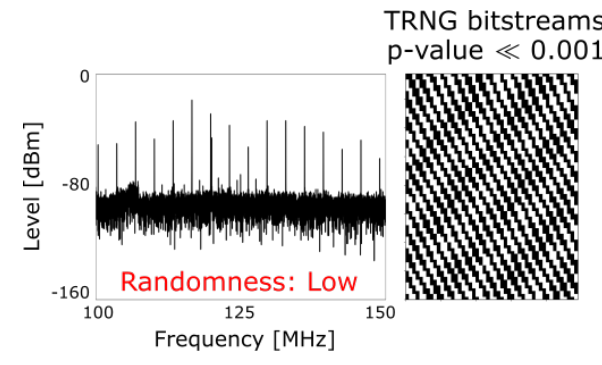

(a) $58.3 \mathrm{MHz}$ injection

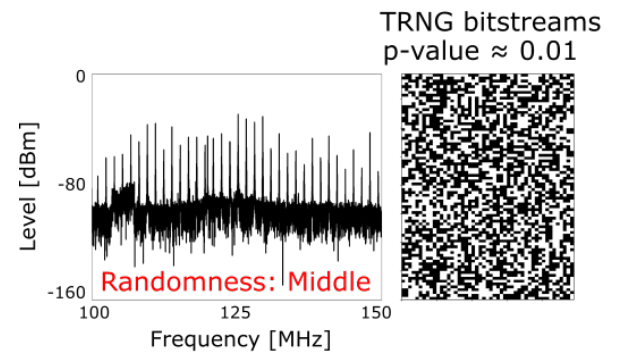

(d) $18.5 \mathrm{MHz}$ injection

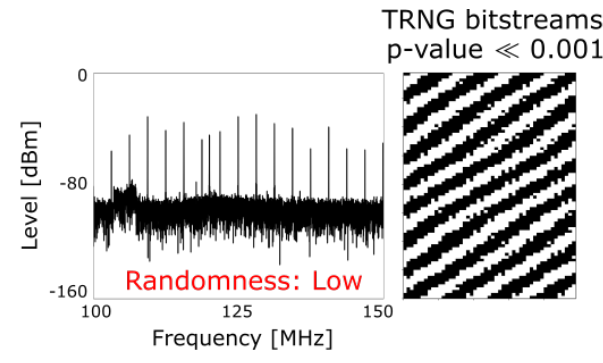

(b) $78.1 \mathrm{MHz}$ injection

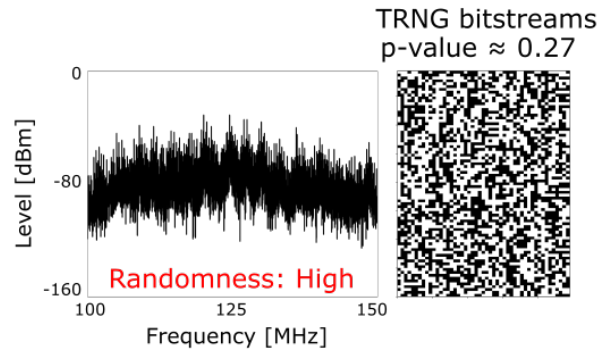

(e) $27.2 \mathrm{MHz}$ injection

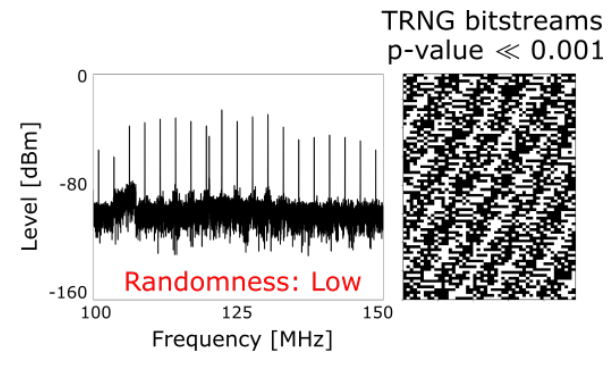

(c) $90.3 \mathrm{MHz}$ injection

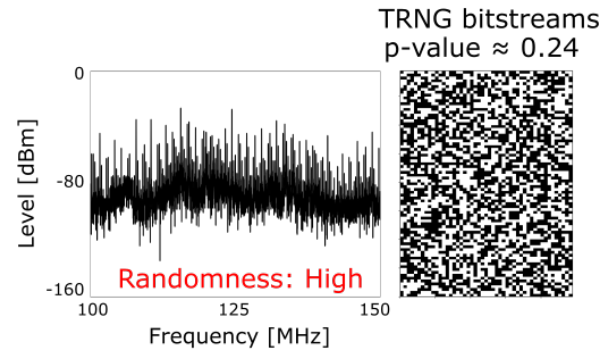

(f) $91.4 \mathrm{MHz}$ injection

Fig. 9. Randomness estimation using side-channel information

Next, we consider how to observe the changes in the internal processing of RO from the common-mode current. In the time domain waveform of Fig. 8, the common-mode current (red lines) was overlaid on Fig. 7. Each common-mode current oscillated at the same frequency as the injection frequency. In other words, the common-mode current largely reflected the influence of the injected sinusoidal waves in the time domain. Therefore, it is effective to observe the injection frequency and other frequency bands separately in the frequency domain to suppress the influence of the injection frequency and more clearly observe the operation of TRNG.

Next, we consider the changes in the frequency spectra when the ROs were locked. The graphs on the right sides of Fig. 7(a) and (b) illustrate the frequency spectra on the left side. Figures 7(a) and (b) can be clearly distinguished, similar to the left figures. A large number of peaks were observed when the ROs were unlocked (Fig. 7(a)) because of the noise caused by the jitter and the RO interactions. In contrast, the noise was reduced, and the number of peaks decreased when the ROs were locked (Fig. 7(b)). Therefore, the changes in the RO states clearly appeared in the frequency domain. Figure 8 shows that these changes can be measured in the same way even in the common-mode current. The difference between the unlocked (Fig. 8 (a)) and locked (Fig. 8 (b)) states was clearly observed for the range of 100 to $150 \mathrm{MHz}$.

This experiment confirmed that the ROs were locked in a narrow frequency range, including $57.0 \mathrm{MHz}$. The ROs can be locked in the range of $10-100 \mathrm{MHz}$ in four frequency ranges (i.e., 56-59 MHz, 77-79 MHz, 90-91 MHz, and 97-98 MHz). These frequency ranges were discontinuous with each other. Thus, a slight frequency range that can lock the ROs must be searched for to lock the ROs. However, the manufacturing variation of the inverter and the circuit structure of the device affect the requirement to lock the ROs; hence, the frequency at which the ROs can be locked is unique to the device. Because of this problem, determining the requirement for locking of RO is difficult without knowing the contents of the device or the output of ROs or TRNG.

In contrast, these experimental results demonstrated that the changes in the internal processing of the ROs can be observed from the common-mode current. Using these results, attacks are possible even if the attacker does not know the requirement to lock the ROs. Presumably, using these changes, we can estimate the state in which the randomness of the TRNG is reduced.

\section{Estimation of the randomness based on common-mode current observation}

This section shows that the degradation of the randomness of the TRNG can be estimated using the common-mode current as the side-channel information based on the previous experimental result.

In this experiment, assuming that the frequency that locks the RO is unknown, sinusoidal waves in the range of 10 to 100 $\mathrm{MHz}$ were induced in the power cable attached to the TRNG. At this time, we observed the common-mode current from the current probe and obtained the waveform in the frequency domain. We estimated that the randomness of the TRNG would be degraded when the obtained waveform in the frequency domain was similar to the waveform shown in Fig. 8(b). We also observed the bit stream generated by the TRNG to evaluate the actual degradation of the randomness.

Figure 9 illustrates the waveform of the common-mode current in the frequency domain, the estimated randomness result, and the bit stream generated by TRNG. This bit stream is displayed as a two-dimensional black and white image corresponding to ones and zeros, respectively, raster scanning left-to-right, then bottom-to-top. The DFT test in the National Institute of Standards and Technology (NIST) test suite [15] was used to evaluate the bit-stream randomness. The statistics 
were analyzed using $6.5 \times 10^{4}$ bits, and the level of significance was set to $0.1 \%$. The randomness of the bit stream was rejected if a P-value is lower than 0.001 .

Figures 9(a) to (c) show the estimated randomness reduction results. Under these experimental conditions, periodicity was observed in the bit stream generated by the TRNG. Moreover, the DFT test rejected the randomness of the generated bit streams. These injection frequencies were included in the frequency ranges (i.e., 56-59 MHz, 77-79 $\mathrm{MHz}$, and 90-91 MHz) that can lock the ROs described in Section 3.B. Similar common-mode current characteristics and degradation of randomness were also observed in the other range (i.e., 97-98 $\mathrm{MHz}$ ).

In contrast, more peaks were observed in Fig. 9(d) than in Figs. 9(a) to (c); hence, the randomness was presumed to be moderate. The P-value calculated in the DFT test exceeded 0.001 in this condition; thus, the randomness was not rejected.

Finally, for the waveforms shown in Figs. 9(e) and (f), we estimated that the randomness was not degraded. The randomness was not rejected because the P-value was considerably higher than 0.001 . These results confirmed that we can use the common-mode current to estimate the states in which the randomness is greatly degraded. In addition to the injection frequencies shown in Fig. 9, the common-mode currents shown in Fig. 8(b) were found at several injection frequencies; however, the ROs were locked in each case.

In this experiment, we demonstrated that estimating the randomness of the TRNG was possible using the commonmode current as side-channel information. Moreover, the randomness of the TRNG can be noninvasively reduced by injecting sinusoidal waves from a distance.

\section{COUNTERMEASURES}

This section describes the possible countermeasures against the proposed attack.

In the attack on the RO-based TRNG conducted in Section 3 , the attack degraded the randomness of the TRNG because it introduced the sinusoidal EM waves at specific frequencies. Unlike a fault injection attack on a cryptographic module, one countermeasure could be to suppress the specific frequencies capable of locking the ROs because this frequency injection attack is effective only at specific frequencies.

For example, it is conceivable that the disturbance frequency that degrades the randomness could be kept out of the IC by dynamically controlling the impedance between it and the board on which the TRNG is mounted [16]. In addition to these countermeasures, a combination of conventional EMC countermeasures, such as ferrite cores, may be able to prevent interference from the multiple frequencies that could be used for the attacks.

\section{CONCLUSION}

This study presented a noninvasive frequency injection attack for degrading the randomness of an RO-based TRNG from a distance using IEMI-based EM wave injection and estimating the randomness from the common-mode current.

In this experiment, the disturbance waves injection and the measurements of the common-mode current, including information, was done approximately 40 and $35 \mathrm{~cm}$ away from the circuit. In contrast, information can be acquired by measuring the emission from equipment at several meters to several tens of meters from the previous research [17-19]. Therefore, leakage information can be acquired at a distance even in device-implemented TRNGs. Moreover, injecting EM waves from a distance is possible based on the results of conventional studies [20-21].

We extracted only the RO-based TRNG and evaluated it to demonstrate the possibility of a remote noninvasive frequency injection attack. However, in an actual device, an RO-based TRNG is mounted in the device as part of the system. A frequency injection attack can be performed from the vicinity of the RO-based TRNG mounted on the smart card [1]. In addition, fault injection using IEMI [5] and electromagnetic analysis using information leakage conducted to external wiring [13] against a cryptographic core mounted on the FPGA has already been presented. Therefore, based on the results obtained earlier, we consider that this method will be effective against an RO-based TRNG, which is part of the system. We also will investigate faster and more accurate estimation methods to estimate the TRNG randomness.

\section{REFERENCES}

[1] A.T. Markettos, S.W. Moore, "The frequency injection attack on ringoscillator-based true random number generators,” CHES, vol. 5747, pp. 317-331, 2009.

[2] P. Bayon, L. Bossuet, A. Aubert, V. Fischer, F. Poucheret, B. Robisson, P. Maurine, "Contactless electromagnetic active attack on ring oscillator based true random number generator," Constructive side-channel analysis and secure design, COSADE, vol. 7275, pp. 151-166, 2012.

[3] P. Bayon, L. Bossuet, A. Aubert, V. Fischer, "Fault model of electromagnetic attacks targeting ring oscillator-based true random number generators,” Journal of Cryptographic Engineering, vol. 6, no. 1, pp. 61-74, 2016.

[4] P. Haddad, C. Kasmi, J.L. Esteves, V. Houchouas, "Electromagnetic harmonic attack on transient effect ring oscillator based true random number generator,” Hardwear.io Conference, Den Haag, Netherlands, 2016.

[5] Y. Hayashi, N. Homma, T. Sugawara, T. Mizuki, T. Aoki, H. Sone, "Non-invasive EMI-based fault injection attack against cryptographic modules," Electromagnetic Compatibility (EMC), 2011 IEEE International Symposium on, pp. 763-767, 2011.

[6] C.R. Paul, "Introduction to Electromagnetic Compatibility," WileyInterscience, New York: John-Wiley \& Sons, 1992.

[7] B. Sunar, W.J. Martin, D.R. Stinson, “A provably secure true random number generator with built-in tolerance to active attacks,” IEEE Trans. Comput, vol. 56(1), pp. 109-119, 2007.

[8] B. Mesgarzadeh, A. Alvandpour, "A study of injection locking in ring oscillators," Proc. IEEE International Symposium on Circuits and Systems, vol. 6, pp. 5465-5468, 2005.

[9] J. Drewniak, F. Sha, T. Van Doren, T. Hubing, J. Shaw, "Diagnosing and modeling common-mode radiation from printed circuit boards with attached cables,” Proc. IEEE Symp. Electromagn. Compat., pp. 465-470, 1995.

[10] D. Hockanson, J. Drewniak, T. Hu Bing, T. Van Doren, F. Sha, M. Wilhelm, "Investigation of fundamental EMI source mechanisms driving common-mode radiation from printed circuit boards with attached cables,” IEEE Trans. on Electromagnetic Compatibility, vol. 38, no. 4, pp. 557-566, 1996.

[11] T.H. Hubing, "Printed circuit board EMI source mechanisms," Proc. IEEE Symp. Electromagn. Compat., vol. 1, pp. 1-3, 2003.

[12] T. Fischer, M. Leone, M. Albach, "An analytical model for studying the electromagnetic radiation of power-bus structures," In IEEE 
International Symposium on Electromagnetic Compatibility, 2003. EMC 2003, vol. 221, pp. 225-230, 2003.

[13] Y. Hayashi, T. Sugawara, Y. Kayano, N. Homma, T. Mizuki, A. Satoh, T. Aoki, S. Minegishi, H. Sone, H. Inoue, "An analysis of information leakage from a cryptographic hardware via common-mode current," EMC'09, pp. 17-20, 2009.

[14] Integrated circuits - Measurement of electromagnetic immunity, 150 $\mathrm{kHz}$ to $1 \mathrm{GHz}$ - Part 3: Bulk current injection (BCI) method IEC 62132-3, 2007.

[15] A. Rukhin, J. Sato, J. Nechvatal, M. Smid, E. Barker, S. Leigh, M Levenson, M. Vangel, D. Banks, A. Heckert, J. Dray, S. Vo, “A statistical test suite for random and pseudorandom number generators for cryptographic applications”, Technical Report SP800-22 Rev. 1a, National Institute of Standards and Technology, USA, 2010.

[16] N. Miura, D. Fujimoto, R. Korenaga, K. Matsuda, M. Nagata, (2014 November). An intermittent-driven supply-current equalizer for $11 \times$ and $4 \times$ power-overhead savings in CPA-resistant 128bit AES cryptographic processor. In Solid-State Circuits Conference (A-SSCC), IEEE.

[17] D. Agrawal, B. Archambeault, R. Rao, P. Rohatgi, "The EM Sidechannel(s),” CHES 2002, Lecture Notes in Computer Science, vol. 2523, pp. 29-45, Aug. 2002.

[18] M. Vuagnoux, S. Pasini, "Compromising electromagnetic emanations of wired and wireless keyboards," Proc. 18th USENIX Security Symposium, 2009, pp. 1-18.

[19] M. Vuagnoux, S. Pasini, “An improved technique to discover compromising electromagnetic emanations," Electromagnetic Compatibility (EMC), 2010 IEEE International Symposium, pp. 121126, 25-30 July 2010.

[20] W.A. Radasky, C.E. Baum, M.W. Wik, "Introduction to the special issue on high-power electromagnetics (HPEM) and intentional electromagnetic interference (IEMI),” IEEE Trans. on Electromagnetic Compatibility, vol. 46, no. 3, pp. 314-321, 2004.

[21] D. Mansson, R. Thottappillil, M. Backstrom, "Propagation of UWB transients in low-voltage power installation networks," IEEE Trans. on Electromagnetic Compatibility, vol. 50, no. 3, pp. 619-629, 2008.

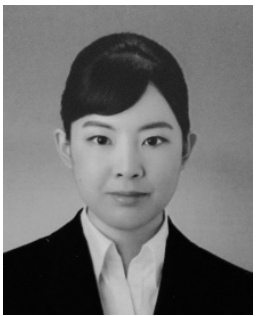

Saki Osuka (S'18) received the B.S. degrees in biology from the University of Tokyo, Tokyo, Japan, in 2017. She is currently working toward the M.E. degree in information sciences at Nara Institute of Science and Technology, Nara, Japan. Her research interests include electromagnetic compatibility and information security.

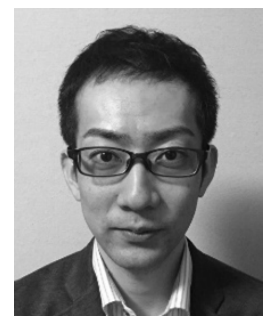

Daisuke Fujimoto (S'12-M'14) received B.E., M.E., and Ph.D. degree from Kobe University, Japan, in 2009, 2011 and 2014, respectively. He is currently an assistant professor in the Graduate School of Information Science, Nara Institute of Science and Technology, Nara, Japan. He is also a visiting assistant professor in the Institute of Advanced Sciences, Yokohama National University. His research interests include hardware security and implementation of security cores. He is a member of IEEE and IEICE.

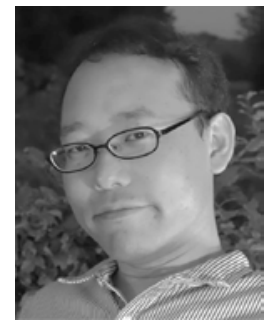

Yu-ichi Hayashi (M'12) received the M.S. and Ph.D. degrees in information sciences from Tohoku University, Sendai, Japan, in 2005 and 2009, respectively. He is currently a Professor in the Graduate School of Information Science, Nara Institute of Science and Technology, Nara, Japan. His research interests include electromagnetic compatibility and information security. Dr. Hayashi is the Chair of EM Information Leakage Subcommittee in IEEE EMC Technical Committee 5.

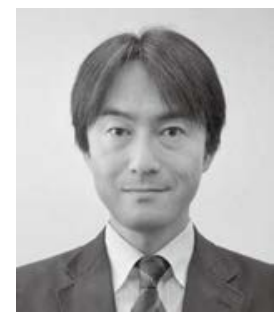

Naofumi Homma (M’01) received the M.S. and Ph.D. degrees in information sciences from Tohoku University, Sendai, Japan, in 1999 and 2001, respectively. He is currently a Professor in the Research Institute of Electrical Communication, Tohoku University. His research interests include hardware security, computer arithmetic, EDA methodology, and cryptographic implementation. Dr. Homma is a member of Advisory Board for Cryptographic Technology, Japan.

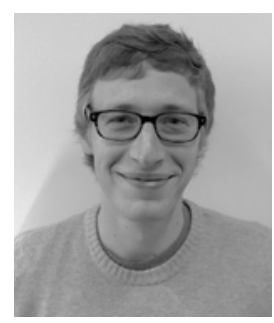

Arthur Beckers received the master's degree in electrical engineering from the KU Leuven, Belgium, in 2015. Since then he is a $\mathrm{PhD}$ candidate with the COSIC research group at KU Leuven. His research interests are fault attacks on cryptographic implementations and side channel analysis.

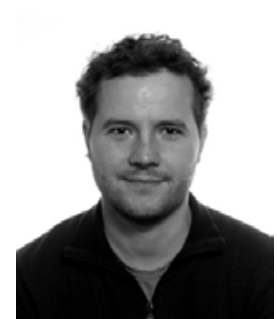

Josep Balasch is a postdoctoral researcher in the research group COSIC at KU Leuven, Belgium. He received a joint Ph.D. degree from KU Leuven and Radboud University Nijmegen in 2014. His research is in the area of embedded security, with main focus on physical attacks (side-channel and fault analysis), countermeasures and cryptographic implementations. He has co-authored more than 25 scientific publications in peerreviewed conferences and journals.

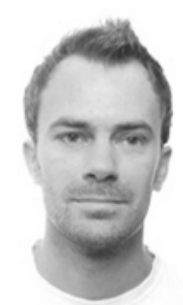

Benedikt Gierlichs is a research expert in the COSIC research group at $\mathrm{KU}$ Leuven in Belgium. He works with the embedded security group and leads the attacks, evaluations and countermeasures team. His research focuses on the (physical) security of embedded devices. Prior to that he was postdoctoral fellow of the Flemish research foundation (Belgium) at COSIC from 2011 to 2017. Benedikt Gierlichs received a MSc degree in IT Security Engineering from the University of Bochum (Germany) in 2006 and a $\mathrm{PhD}$ degree from the electrical 
engineering department of the KU Leuven (Belgium) in 2011. Dr. Gierlichs has co-authored more than 45 scientific publications in peer-reviewed, international conferences and journals. He has served on more than 25 program committees of international conferences and has co-chaired 4 of them.

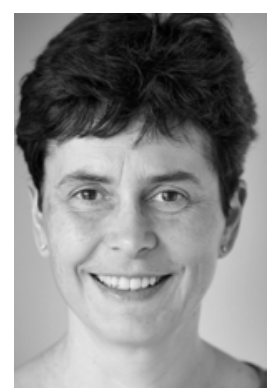

Ingrid Verbauwhede is a Professor in the research group COSIC of the EE Department of the KU Leuven in Belgium. At COSIC, she leads the embedded systems and hardware group. She is also adjunct professor at the EE department at UCLA, Los Angeles, CA. She is a Fellow of IEEE and a Member of IACR. She was elected as member of the Royal Academy of Belgium for Science and the Arts in 2011. She received an ERC Advanced Grant in 2016 and the IEEE Computer Society Technical Achievement Award in 2017. The list of Prof. Verbauwhede's publications and patents is available at www.esat.kuleuven.be/cosic. 